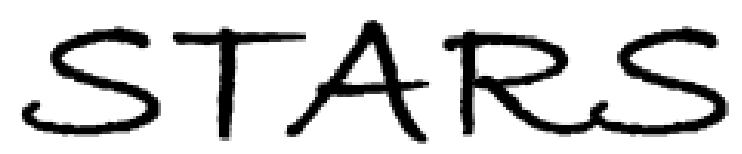

University of Central Florida

STARS

$1-1-2014$

\title{
Micro electro mechanical cantilever with electrostatically controlled tip contact
}

\author{
Imen Rezada \\ University of Central Florida \\ Javaneh Boroumand \\ University of Central Florida \\ Evan M. Smith \\ University of Central Florida \\ Robert E. Peale \\ University of Central Florida
}

Find similar works at: https://stars.library.ucf.edu/facultybib2010 University of Central Florida Libraries http://library.ucf.edu

This Article is brought to you for free and open access by the Faculty Bibliography at STARS. It has been accepted for inclusion in Faculty Bibliography 2010 s by an authorized administrator of STARS. For more information, please contactSTARS@ucf.edu.

\section{Recommended Citation}

Rezada, Imen; Boroumand, Javaneh; Smith, Evan M.; and Peale, Robert E., "Micro electro mechanical cantilever with electrostatically controlled tip contact" (2014). Faculty Bibliography 2010s. 5995. https://stars.library.ucf.edu/facultybib2010/5995

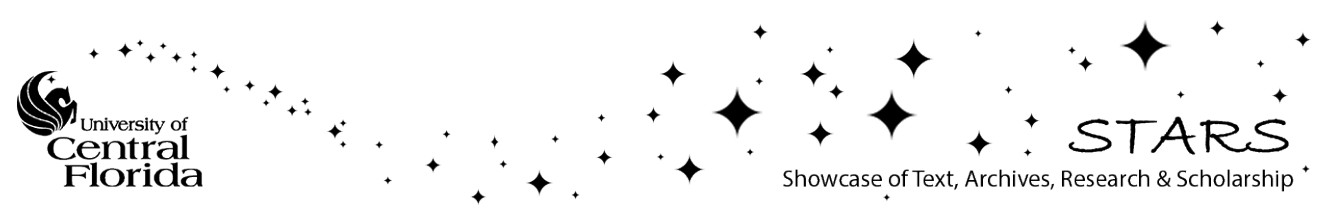




\section{Micro electro mechanical cantilever with electrostatically controlled tip contact}

Cite as: Appl. Phys. Lett. 105, 033514 (2014); https://doi.org/10.1063/1.4891496

Submitted: 22 February 2014 . Accepted: 16 July 2014 . Published Online: 25 July 2014

Imen Rezadad, Javaneh Boroumand, Evan M. Smith, and Robert E. Peale
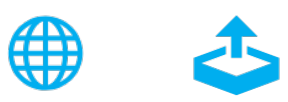

View Online

\section{ARTICLES YOU MAY BE INTERESTED IN}

Experimental investigation of resonant MEMS switch with ac actuation

Applied Physics Letters 108, 253501 (2016); https://doi.org/10.1063/1.4954262

Active MEMS metamaterials for THz bandwidth control

Applied Physics Letters 110, 161108 (2017); https://doi.org/10.1063/1.4980115

Mechanical behavior simulation of MEMS-based cantilever beam using COMSOL multiphysics AIP Conference Proceedings 1653, 020005 (2015); https://doi.org/10.1063/1.4914196

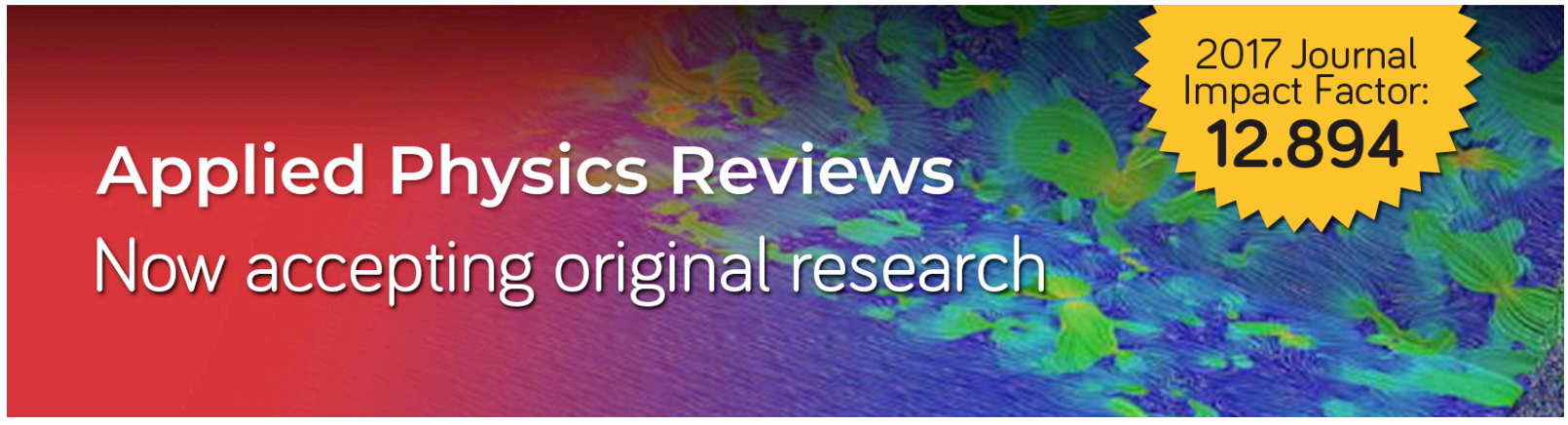




\title{
Micro electro mechanical cantilever with electrostatically controlled tip contact
}

\author{
Imen Rezadad, Javaneh Boroumand, Evan M. Smith, and Robert E. Peale \\ Physics Department, University of Central Florida, Orlando, Florida 32816, USA
}

(Received 22 February 2014; accepted 16 July 2014; published online 25 July 2014)

\begin{abstract}
A micro-electro-mechanical system (MEMS) cantilever that lifts from the surface by electrostatic force is described. The design is composed of three conductors: a fixed buried plate, a fixed surface plate, and a moveable cantilever. All have the same square shape and are arranged parallel in a vertical stack with aligned edges. The surface plate and cantilever are biased at the same potential, and the buried plate is oppositely biased. Theoretical analysis based on values of position-dependent coefficients of capacitance and electrostatic induction from finite element method demonstrates the sign of the force on the cantilever and determines its magnitude. Video microscopy and electrical measurements demonstrate the electrostatic lifting of the cantilever in a fabricated MEMS device. The vertical displacement of the cantilever is quantified from changes in optical interference fringes, and the displacement magnitude agrees with expectations based on estimated strengths of upward electrostatic force and downward elastic restoring force. (C) 2014 AIP Publishing LLC.
\end{abstract}

[http://dx.doi.org/10.1063/1.4891496]

Edwards ${ }^{1}$ proposed a micro-electro-mechanical system (MEMS) infrared (IR) detector composed of a cantilever, a surface plate, and a buried plate. Lifting of the cantilever tip from a surface contact pad by electrostatic force is an essential principle of operation, in which the duty cycle of a repetitively opened and closed tip contact is a measure of the absorbed infrared energy. ${ }^{2}$ This space-efficient three-layer design allows high fill factors. We confirm by calculation and experiment that an electrostatic force of the required direction and of sufficient magnitude is achieved. Many groups have considered electrostatic positioning control of MEMS devices [e.g., Refs. 3-12], but Edwards's design makes higher fill factor possible and suggests broader applications as means of overcoming stiction in MEMS switches, actuators, and micromirrors. ${ }^{13,14}$

The net force on the cantilever is determined from the position dependence of the coefficients of capacitance and electrostatic induction. The model system consists of 3 parallel plates (Fig. 1, lower inset), which are assumed square and each with area $A$. A buried plate (1) is at depth $d$ below the surface and is held at a potential of $-V / 2$. A fixed surface plate (2) is held at potential $+V / 2$. The cantilever (3) is a variable height $z$ above the surface plate, to which it is electrically connected so that its potential is also $+V / 2$. The energy of a system of conductors at fixed potentials $\phi_{i}$ is ${ }^{15}$

$$
U=\frac{1}{2} \sum_{a, b} C_{a b} \phi_{a} \phi_{b}
$$

The $C_{a b}(a \neq b)$ are the coefficients of electrostatic induction $\left(C_{a b}=C_{b a}\right)$, and the $C_{a a}$ are the coefficients of capacity. The former are always negative and the latter are always positive. Both coefficient types depend on the conductor shapes, sizes, and relative positions. For our model system, the energy is

$$
U=\frac{V^{2}}{8}\left(-2 C_{12}-2 C_{13}+2 C_{23}+C_{11}+C_{22}+C_{33}\right)
$$

Differentiation of this energy with respect to the vertical position $z$ of the cantilever gives the electrostatic force on that plate

$$
\begin{aligned}
F & =+\frac{\partial U}{\partial z} \\
& =\frac{V^{2}}{8}\left[-2 \frac{\partial C_{12}}{\partial z}-2 \frac{\partial C_{13}}{\partial z}+2 \frac{\partial C_{23}}{\partial z}+\frac{\partial C_{11}}{\partial z}+\frac{\partial C_{22}}{\partial z}+\frac{\partial C_{33}}{\partial z}\right] .
\end{aligned}
$$

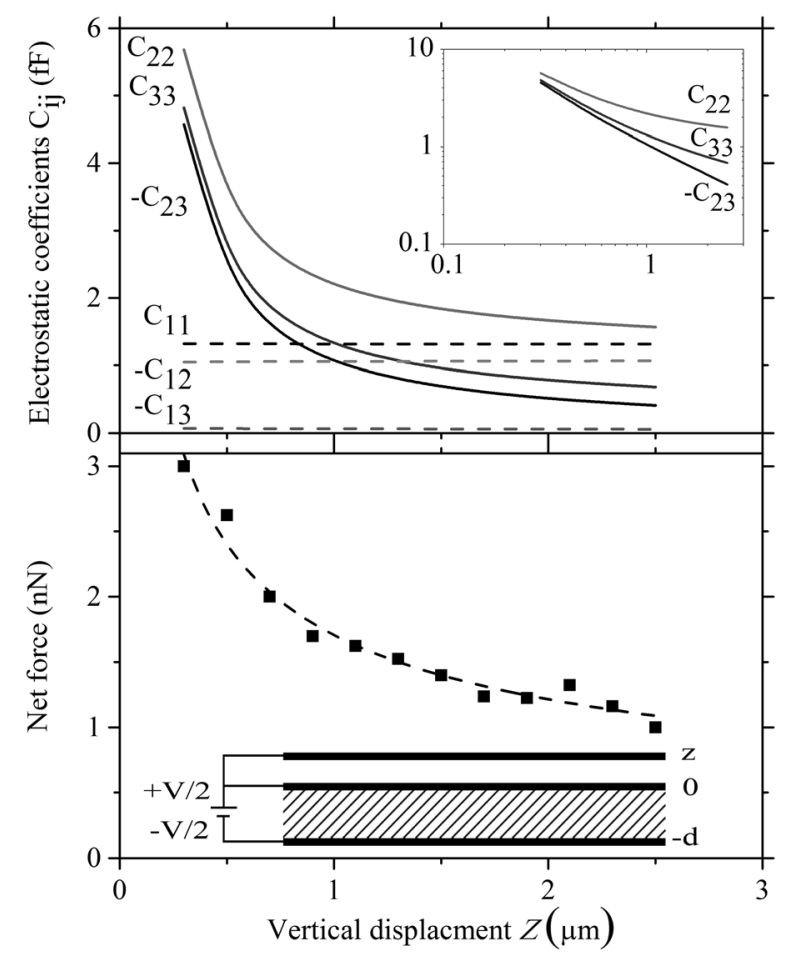

FIG. 1. (upper) Coefficients $C_{i j}$ for system of three parallel square plates as a function of the cantilever height $\mathrm{z}$ for plate area $10 \mu \mathrm{m} \times 10 \mu \mathrm{m}$. Inset: $\log -\log$ plot for three of the curves. (lower) Net force on $10 \mu \mathrm{m} \times 10 \mu \mathrm{m}$ cantilever vs. its height above the surface for $20 \mathrm{~V}$ bias. Symbols are calculation results. The line is a fit to $\frac{1}{\sqrt{z}}$. Inset: Model schematic. 
It is very important that the " + " appears before the derivative in Eq. (3), rather than the usual "-" from ordinary mechanics. The quantity $U$ is the electric energy of the plates alone, and it does not include the energy of the large charge reservoirs, batteries, or power supplies that are necessary to maintain the plates at constant potential as the cantilever moves. These charge sources or sinks do work in moving charges to maintain the potentials, so their energy changes. When this is properly included, it turns out that it is the positive derivative of $U$ that determines the force. ${ }^{15}$

Fig. 1 (upper) plots the six $z$-dependent coefficients calculated by finite element method (FEM) (FastCap ${ }^{16}$ and Elmer ${ }^{17,18}$ ) for $10 \mu \mathrm{m} \times 10 \mu \mathrm{m}$ plates. As $\mathrm{z}$ increases, the magnitude of $C_{13}$ decreases due to fringe-field weakening, which lessens induced charges. Generally, however, the z-dependence of all the $C_{1 \mathrm{j}}$ is very weak, because the surface plate screens the buried plate from the field of the cantilever, whose motion therefore has little effect on the buried plate's total charge. This allows us to ignore the derivatives of those three coefficients in Eq. (3), giving

$$
F \cong \frac{V^{2}}{8}\left[2 \frac{\partial C_{23}}{\partial z}+\frac{\partial C_{22}}{\partial z}+\frac{\partial C_{33}}{\partial z}\right]
$$

The induction coefficient $C_{23}$ (which is negative) approaches zero with increasing separation of the two upper conductors, as expected, so that $\mathrm{d} C_{23} / \mathrm{d} z$ is positive. The positive coefficients of capacity $C_{22}$ and $C_{33}$ are expected to decrease to constant positive values as the separation between the top two conductors increases, and we expect $C_{22}>C_{33}$ because the surface plate is near to two plates while the cantilever is near to just one. These expectations are also confirmed in Fig. 1, upper. Thus the $z$ derivatives of these coefficients of capacity are negative. The inset in Fig. 1 upper presents a log-log plot of the three coefficients in Eq. (4). The slope of $-C_{23}$ is more negative than the slopes of the other two, so that the first term in Eq. (4) exceeds the sum of the magnitudes of the other two terms. Hence, the force is positive. In other words, the direction of the force is the same as if the cantilever is being repelled from the surface. (We eschew the convenience of phrases such as "repulsive force" and "repelled by the surface" to avoid conceptual controversies associated with the fiction of force at a distance. Each conductor feels only the negative pressure due to the fields at its own surface. ${ }^{15}$ Integration of this pressure over the surface gives the net force ${ }^{18}$ and confirms the sign found here.)

Fig. 1 (lower) plots the calculated force (Eq. (4)) using coefficient values from Fig. 1 (upper). The net force is positive in the considered range $0.25 \mu \mathrm{m}<z<2.5 \mu \mathrm{m}$, which are the motional limits in the experimental device. The force decreases as the separation increases. Over the range considered, the force is adequately described by a power law. The line shows a fit to the function $\frac{1}{\sqrt{z}}$. That line reveals a small oscillation with $z$ in the force data, which is an artifact due to meshing, as determined using higher mesh density at the expense of longer calculation times. When the permittivity of the structural oxide in the actual device is included, ${ }^{18}$ the force magnitude increases nearly four-fold in comparison to that presented in Fig. 1 for the simple model system.
Without a surface plate, the force on the cantilever would be downward toward the oppositely biased buried plate. On the other hand, if the surface plate were much larger than the others, it would screen the buried plate so that there would be no fields from it at the cantilever, and hence no force on the cantilever. As found above, the force is upwards for plates of equal dimensions. Thus, were the surface plate to increase in size monotonically from zero, the force would change from downward to upwards before decreasing again to zero. There will be an optimum surface plate size that maximizes the upward force. Optimization by 2 dimensional FEM calculations is presented in Ref. 18, which also presents visualization and discussion of the fringing fields.

We fabricated devices described in Ref. 1, which are similar to the simplified model analyzed above. The structural material of the cantilever and the dielectric between surface and buried plates was plasma-enhanced chemical vapor deposited (PECVD) oxide. Buried and surface plates were $100 \mathrm{~nm}$ thick Cr. Cantilever metal was $10 \mathrm{~nm}$ thick $\mathrm{Cr}$ coated with $30 \mathrm{~nm} \mathrm{Au}$, which was electrically connected to the surface plate via $10 \mu \mathrm{m}$ wide arms and anchors. The processing is described in Refs. 19 and 20. Plates of $100 \mu \mathrm{m}$ $\times 100 \mu \mathrm{m}, 50 \mu \mathrm{m} \times 50 \mu \mathrm{m}$, and $20 \mu \mathrm{m} \times 20 \mu \mathrm{m}$ were fabricated. All show the same effects. Fig. 2 is a scanning electron microscopy (SEM) image of one device in its unbiased "null position." Breaking of contact between tip metal and tip pad is electrically sensed. The device differs from the model, as necessary for intended function, ${ }^{1}$ by the presence of dielectrics, an infinite (wafer scale) buried plate, nonparallel flexible cantilever orientation and motion, arms, release holes, electrical traces, and bond pads.

Video microscopy dramatically reveals the upward displacement due to the electrostatic force. Fig. 3 (upper) presents video frames before and after reaching $40 \mathrm{~V}$ applied bias, where the electrostatic force has ripped the cantilever from its anchors, displacing it. Some videos show the cantilever flying away when the anchors give way.

Fig. 3 (lower) presents images of incompletely released cantilevers stuck in polyimide residue. When biased, the cantilever slowly peeled up from the surface. Loss of contact between cantilever and residue is revealed by intrusion of air under the cantilever from the edges and release holes. When

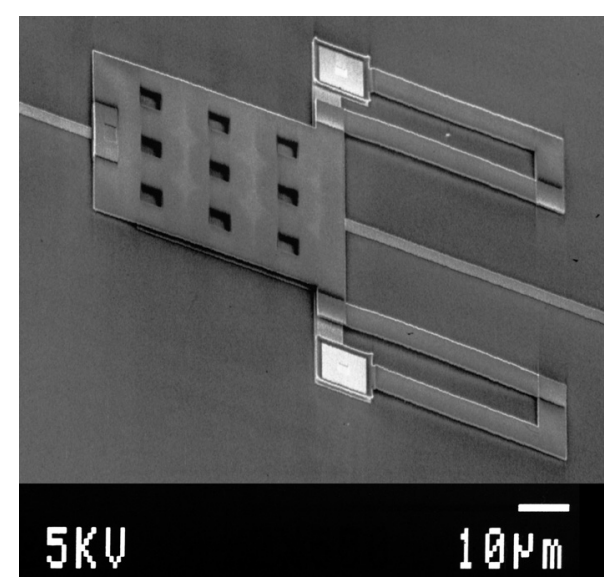

FIG. 2. SEM image of the MEMS cantilever with $50 \mu \mathrm{m} \times 50 \mu \mathrm{m}$ paddle. 

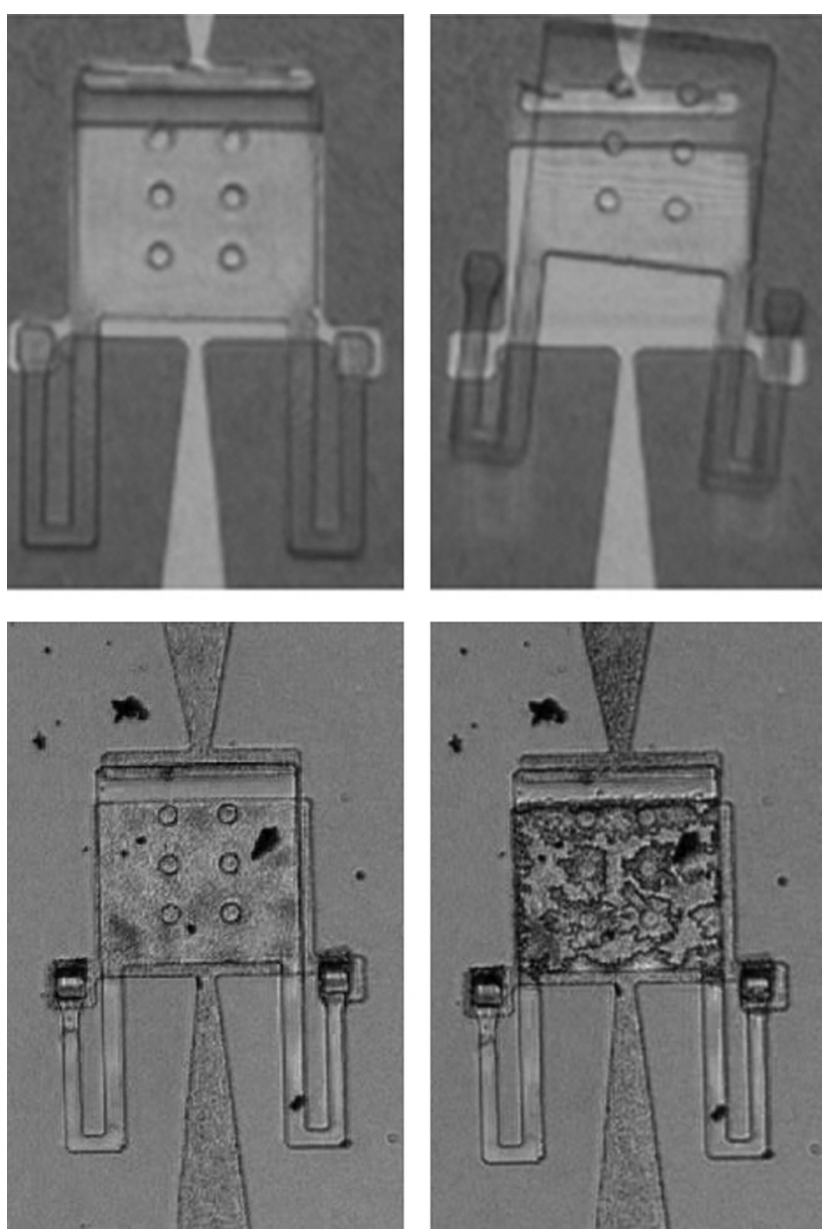

FIG. 3. Video microscopy frames of well-released cantilever before (upper left) and after (upper right) reaching $40 \mathrm{~V}$ applied bias, where upward electrostatic force has ripped the cantilever off its anchors. Video frames for incompletely released cantilever before (lower left) and after (lower right) applying bias. Electrostatic force lifts the cantilever from polyimide residue, causing air bubbles to intrude under the semi-transparent cantilever from the edges and release holes.

the bias is removed, the cantilevers sink back into the sticky film, and the air is squeezed back out.

Vertical displacement of the semitransparent cantilever was quantified by an optical interferometry method described in Ref. 19 on a large cantilever with $100 \mu \mathrm{m}$ $\times 100 \mu \mathrm{m}$ paddle using a 600 -nm-wavelength long-pass filter to improve contrast. At zero bias, the highest density of fringes occurs near the middle of the paddle, where the curvature of the paddle is evidently maximum. An SEM image of one of these large cantilevers confirms this interpretation of the initial paddle deformation in null position. ${ }^{18}$ When bias is applied, the fringes from the middle of the paddle are observed to shift toward the tip, increasing their spacing, while no change in the interference pattern is observed near the base of the paddle or arms. This indicates a lifting of the tip and flattening of the paddle with bias. Fig. 4 plots the height of the gap between cantilever and surface determined from the first several dark fringes nearest the tip where their visibility is highest. We assume that the dark fringe nearest the tip at $0 \mathrm{~V}$ has a quarter-wave gap of $150 \mathrm{~nm}$. Insets are microscope images at 20 and $40 \mathrm{~V}$, where the difference in interference pattern is most obvious over the rightmost release holes which change from bright to dark. The observed

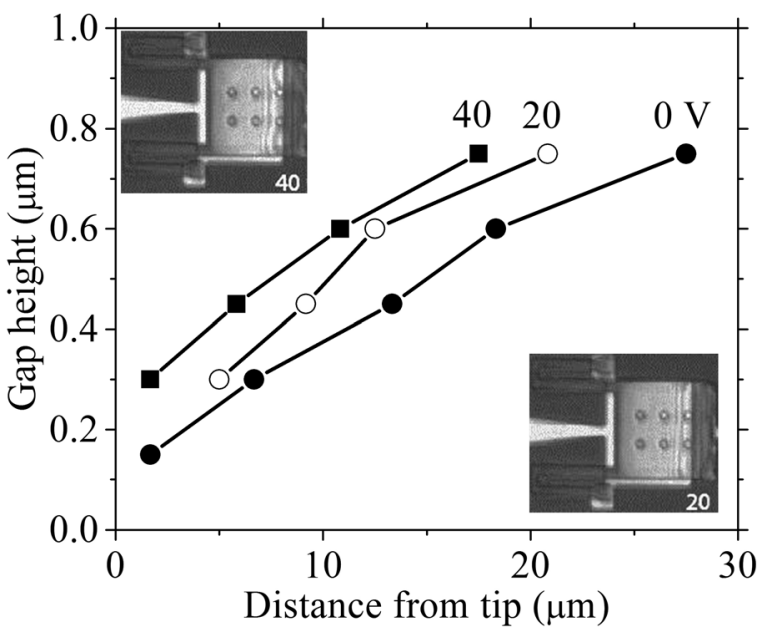

FIG. 4. Height of gap between surface plate and cantilever as function of distance from tip for three values of applied bias. Insets show images with different interference patterns in red light at $20 \mathrm{~V}$ and $40 \mathrm{~V}$.

average change in height with bias is roughly $5 \mathrm{~nm} / \mathrm{V}$. An estimate of the spring constant for bending of the paddle due to a concentrated force near the tip ${ }^{21}$ is $0.22 \mathrm{~N} / \mathrm{m}$. Thus, to obtain $100 \mathrm{~nm}$ of tip lift for $20 \mathrm{~V}$ bias requires a force of $\sim 22 \mathrm{nN}$. We note that the portion of the large curved cantilever feeling most of the lifting and paddle-flattening force is evidently near the tip, a strip of say $\sim 5 \mu \mathrm{m} \times 100 \mu \mathrm{m}$, which is five-fold larger than the $10 \mu \mathrm{m} \times 10 \mu \mathrm{m}$ area of the model cantilever in Fig. 1 . We also note that the structural oxide tends to increase the electrostatic force. ${ }^{18}$ Hence, the observed displacement agrees with expectations in order of magnitude.

Setting the electrostatic force, which for the simple model goes as $\sim \frac{V^{2}}{\sqrt{2}}$ according to Eq. (4) and Fig. 1, equal to the elastic force, which goes as $z$, we expect the displacement to increase as $V^{4 / 3}$. In fact, Fig. 4 suggests that displacement depends sub-linearly on $V$. We note that some cantilevers are destroyed at $40 \mathrm{~V}$ bias by excessive leakage

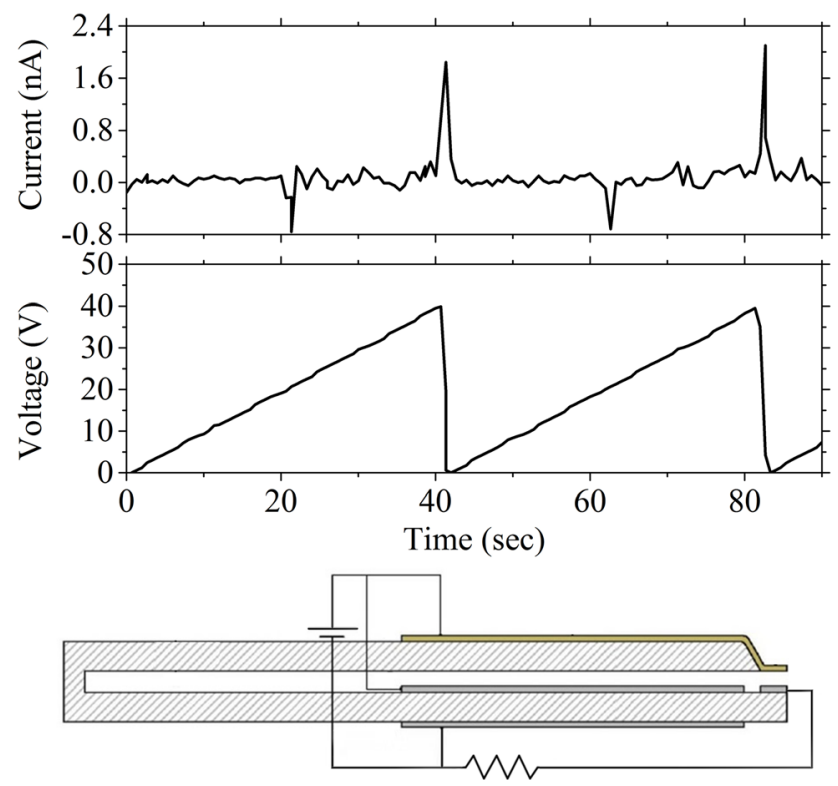

FIG. 5. (top) Applied sawtooth ramp bias applied between cantilever and buried plate and measured current through load resistor. (bottom) Schematic of device with external circuitry. 
current between surface and buried plates. Any leakage reduces the expected electrostatic force on the plates. Leakage can be reduced by using materials with larger dielectric constant.

The vertical displacement caused by the electrostatic force was also observed electrically. In null position, a bias applied to the cantilever should appear across the load resistor shown schematically in Fig. 5. When contact with the tip breaks due to the lifting of the cantilever, the voltage across the load returns to $0 \mathrm{~V}$. A saw tooth ramp bias was applied as shown in Fig. 5. The actual tip contact resistance was very high (due to residue or curling), so that no direct current was observed in null position. Instead, as bias increased, breaking of physical contact at the tip caused a sudden redistribution of charge, which was sensed as a small current in the load resistor. When the bias was switched off, there appeared an induced current in the load of the opposite sign, which we interpret as being due to the sudden return of the cantilever to null position. This effect is repeatable.

The sign and relative size of the current spikes in Fig. 5 are easily explained. When the positively biased tip is in physical (but not electrical) contact with the tip pad, the latter is negatively charged by induction. When the cantilever pops up, some of this negative charge flows away through the load resistor, causing negative current. The cantilever continues to rise slowly during the ramp, allowing more negative charge to bleed off, but the rate of this charge flow is below the noise. When the bias is shut off, the cantilever returns suddenly to null position from its maximum height, inducing a large positive current as all of the original negative charge rushes back up through the load resistor to inductively recharge the tip pad.

The tip may be designed so that the electrostatic force overcomes the Casimir sticking force even for very close electrical contact between tip and tip pad. ${ }^{18}$ Noise equivalent power and noise equivalent temperature difference for IR sensing mechanism are discussed in Ref. 21. In summary, an electrostatic force that lifts a MEMS cantilever from the surface, for a design composed of three parallel conducting plates, has been demonstrated theoretically and experimentally.

This project was supported in part by the Florida High Technology Corridor (I-4) Program.

${ }^{1}$ O. Edwards, U.S. patent 7,977,635 B2 (July 12, 2011).

${ }^{2}$ E. Smith, J. Boroumand, I. Rezadad, P. Figueiredo, J. Nath, D. Panjwani, R. E. Peale, and O. Edwards, Proc. SPIE, Infrared Technology and Applications XXXIX 8704, 87043B (2013).

${ }^{3}$ H. Ren, F. Tao, W. Wang, and J. Yao, J. Micromech. Microeng. 21, 045019 (2011).

${ }^{4}$ T. Sugimoto, K. Nonaka, and M. N. Horenstein, J. Microelectromech. Syst. 14, 718 (2005).

${ }^{5}$ S. He and R. B. Mrad, IEEE Trans. Ind. Electron. 52, 974 (2005).

${ }^{6}$ Y. Suzuki, D. Miki, M. Edamoto, and M. Honzumi, J. Micromech. Microeng. 20, 104002 (2010).

${ }^{7}$ F. Hu, W. Wang, and J. Yao, J. Micromech. Microeng. 21, 115029 (2011). ${ }^{8}$ S. He and R. B. Mrad, J. Microelectromech. Syst. 17, 532 (2008).

${ }^{9}$ W. Wang, F. Tao, Q. Wang, C. Qiu, Z. Chen, and J. Yao, Proc. SPIE 8617, 861702 (2013).

${ }^{10}$ X. M. Zhang, F. S. Chau, C. Quan, Y. L. Lam, and A. Q. Liu, Sens. Actuators, A 90, 73 (2001).

${ }^{11}$ S. W. Chyuan, Y. S. Liao, and J. T. Chen, J. Microelectromech. Syst. 14, 305 (2005).

${ }^{12}$ J. Yao, F. Hu, D. Cai, and W. Jiang, Proc. SPIE 7209, 72090K (2009).

${ }^{13}$ S. He and B. Mrad, Proc. SPIE 5264, 154 (2003).

${ }^{14}$ F. Hu, J. Yao, C. Qiu, and H. Ren, J. Electrostat. 68, 237 (2010).

${ }^{15}$ L. D. Landau and E. M. Lifshitz, Electrodynamics of Continuous Media, 2nd ed. (Elsevier Butterworth Heinemann, Amsterdam, 1984).

${ }^{16}$ FastFieldSolvers S.R.L, Via de Castillia, 7, 20871 Vimercate (MB) Italy.

${ }^{17}$ CSC - IT Center for Science Ltd., P.O. Box 405, FI-02101 Espoo, Finland.

${ }^{18}$ I. Rezadad, J. Boroumand Azad, E. M. Smith, A. Alhasan, and R. E. Peale, Proc. SPIE, Infrared Technology and Applications XL 9070, 90701U (2014).

${ }^{19}$ I. Rezadad, J. Boroumand, E. Smith, P. Figueiredo, and R. E. Peale, MRS Proceedings 1536, 155 (2013).

${ }^{20}$ J. B. Azad, I. Rezadad, J. Nath, E. M. Smith, and R. E. Peale, Proc. SPIE 8682, 868226 (2013).

${ }^{21}$ J. Boroumand Azad, I. Rezadad, E. M. Smith, A. Alhasan, and R. E. Peale, Proc. SPIE, Infrared Technology and Applications XL 9070, 907020 (2014). 